C2016, Elsevier. Licensed under the Creative Commons Attribution-NonCommercialNoDerivatives 4.0 International http://creativecommons.org/about/downloads 


\section{Effects of etching time on alpha tracks in Solid State Nuclear Track Detectors.}

Gavin Gillmore*, David Wertheim and Simon Crust.

Faculty of Science, Engineering and Computing, Kingston University, Surrey, UK, KT1 2EE.

E-mail: G.Gillmore@kingston.ac.uk, D.Wertheim@kingston.ac.uk.

*Corresponding author.

\section{Abstract}

Solid State Nuclear Track Detectors (SSNTDs) are used extensively for monitoring alpha particle radiation, neutron flux and cosmic ray radiation. Radon gas inhalation is regarded as being a significant contributory factor to lung cancer deaths in the UK each year. Gas concentrations are often monitored using CR39 based SSNTDs as the natural decay of radon results in alpha particles which form tracks in these detectors. Such tracks are normally etched for about 4 hours to enable microscopic analysis. This study examined the effect of etching time on the appearance of alpha tracks in SSNTDs by collecting 2D and 3D image datasets using laser confocal imaging techniques. Etching times of 2 to 4 hours were compared and marked differences were noted in resultant track area. The median equivalent diameters of tracks were $20.2,30.2$ and $38.9 \mu \mathrm{m}$ for etching at 2,3 and 4 hours respectively. Our results indicate that modern microscope imaging can detect and image the smaller size tracks seen for example at 3 hours etching time. Shorter etching times may give rise to fewer coalescing tracks although there is a balance to consider as smaller track sizes may be more difficult to image. Thus etching for periods of less than 4 hours clearly merits 
further investigation as this approach has the potential to improve accuracy in assessing the number of tracks.

\section{Keywords.}

Etching time, SSNTDs, alpha tracks, radon.

\section{Introduction.}

Solid State Nuclear Track Detectors (SSNTDs) are used extensively to provide data on various types of charged particles (Mozzo et al., 1996), and therefore used in neutron detection and dosimetry (Palacios et al., 2011), cosmic-ray physics (Tommasino, 2004) and medical applications such as boron neutron capture therapy (Smilgys et al., 2013). SSNTDs can also be used to monitor radon concentrations (Phillips et al., 2004) in the home and workplace.

Exposure to radon gas $\left({ }^{222} \mathrm{Rn}\right)$ and associated ionising decay products can cause lung cancer in humans (Darby et al., 2005) and it is thought to be the cause of about 1100 lung cancer related deaths each year in the UK (Gray et al., 2009). In the USA the Environmental Protection Agency (EPA) suggest that around 21,000 lung cancer deaths per year (with 160,000 deaths linked to smoking) are the result of exposure to radon. Of this number 2,900 occur among people who have never smoked, and hence it is viewed by the EPA as the number one cause of lung cancer among non-smokers (see News Release, January $13^{\text {th }} 2005$, Surgeon General National Advisory on Radon). The work of Darby et al. (2005) and others also highlights the fact that there is a synergistic effect between radon and smoking. The EPA note that a never smoker exposed to around $48 \mathrm{~Bq} \mathrm{~m}^{-3}(1.3 \mathrm{pCi} / \mathrm{L})$ of radon has a 2 in 1,000 
chance of lung cancer. A smoker on the other hand also exposed to radon has a 20 in 1,000 chance of dying from lung cancer.

Alpha particles from the decay of radon form sub- microscopic tracks in the detectors. In order to allow these latent tracks to be seen with optical microscope techniques (Durrani, 1997) they must be enlarged by etching (either chemical or electrochemical). From the track density per unit of time of being irradiated, the particle fluence on the SSNTD can be assessed. Track density will provide information on for example radon concentration in the atmosphere, but this may not be enough information for the desired application. An assessment of the energies of the incident particles may be required to for example, reconstruct proton energy spectra, which can be related to neutron energy spectra.

A variety of etchants can be used in the etching process such as potassium hydroxide and sodium hydroxide. We have used a caustic soda solution. Some others have added alcohol to their solution which increases the etching speed, but it also decreases the detector sensitivity (Durrani, 1997; Tommasino, 1997). Tommasino (1997) concluded that the important factors in controlling etching speed were temperature and the concentration of the etching fluid.

Currently etched detectors are usually assessed with optical microscopy and 2D surface image analysis and counted with semi-automatic / image analysis -based techniques (see Nikezic and Yu, 2015). 
We have previously shown that confocal microscopy can be used for non-destructive 3D visualisation of etched SSNTDs (Wertheim et al., 2010a,b; Wertheim and Gillmore, 2014). In these studies we observed that adjoining tracks can coalesce into non-circular areas which require distinction from artefact and hence might affect assessment accuracy. There have been few studies of the effect of etching on track appearance in 2D and by destructive cutting of the detectors. Examples include Dörschel et al. (1996; 1997) who broke SSNTDs to reveal lateral images of tracks for direct measurement. The assessment of the tracks was carried out using a Nikon microscope with the LUCIA image analysis system. Ng et al. (2007) polished the sides of SSNTDs to reveal cross-section of tracks, and then imaged them using a digital camera processing the image with ImageJ. Replicas of tracks were then made using an epoxy resin and assessed using a profilometer.

Aim.

The aim of the study was to examine the effect of etching time on the 2D and 3D appearance of alpha tracks in SSNTDs using confocal microscopy.

\section{Methods.}

Six SSNTDs were placed in a chamber with a radium dial watch for a fixed period (see Figure 1). The detectors were etched for either 2,3 or 4 hours using $6 \mathrm{M} \mathrm{NaOH}$ at a temperature of $90^{\circ} \mathrm{C}$. Thus there were two detectors for each etching time. 
We studied CR39 SSNTDs as they are widely used in charged particle concentration monitoring and are considered to exhibit more similar characteristics in comparison with human tissue than some other types of SSNTDs.

A 'LEXT' OLS4100 confocal laser scanning microscope (Olympus Corporation, Japan) was used to acquire 2D and 3D image datasets of CR-39 plastic SSNTDs. Confocal microscope 3D images were acquired using a x50 objective lens (NA 0.95) with a scan area of 260 by 260 $\mu \mathrm{m} .2 \mathrm{D}$ images were acquired using $\times 10$ and $\times 20$ objective lenses as single images or stitched panoramic images to cover most of the detector surface.

Data were saved as images and also spreadsheet files with height measurements. Software was written with MATLAB (The MathWorks Inc, Natick, MA, USA) in order to analyse the number, area and shape of the tracks. In brief the colour 2D images were converted to binary images such that the tracks could be detected. The shape was determined by a calculation of roundness that is $\left(4 \pi \times\right.$ Area)/ $\left(\right.$ Perimeter $\left.^{2}\right)$; detected shapes with a roundness $\geq 0.67$ were assumed to be approximately circular and hence single tracks. Nine nonoverlapping 2D images were analysed for one detector for each of the three etching times. The images chosen were in equivalent parts of the central region of the detectors and obtained using the $x 20$ objective lens.

\section{Results.}

Figures 2 to 3 show examples of 2D and 3D imaging of tracks in SSNTDs which were etched for either 2, 3 or 4 hours. 
Table 1 summarises the median and interquartile range of track areas and equivalent diameters as well as the number of single circular tracks detected. The total number of tracks for each detector are shown in the lower part of the table; this includes angled as well as coalescing tracks.

\begin{tabular}{|c|c|c|c|}
\hline & 2 hours & 3 hours & 4 hours \\
\hline \multicolumn{4}{|l|}{$\begin{array}{l}\text { Area circular tracks } \\
\left(\mu \mathrm{m}^{2}\right)\end{array}$} \\
\hline median & 320 & 716 & 1186 \\
\hline lower quartile & 259 & 539 & 904 \\
\hline upper quartile & 377 & 848 & 1293 \\
\hline $\begin{array}{l}\text { number of circular } \\
\text { tracks (from } 9 \text { images) }\end{array}$ & 177 & 180 & 177 \\
\hline \multicolumn{4}{|l|}{$\begin{array}{l}\text { Equivalent diameter } \\
\text { circular tracks }(\mu \mathrm{m})\end{array}$} \\
\hline median & 20.2 & 30.2 & 38.9 \\
\hline lower quartile & 18.2 & 26.2 & 33.9 \\
\hline upper quartile & 21.9 & 32.9 & 40.6 \\
\hline $\begin{array}{l}\text { number of circular } \\
\text { tracks (from } 9 \text { images) }\end{array}$ & 177 & 180 & 177 \\
\hline $\begin{array}{l}\text { number of tracks } \\
\text { (from } 9 \text { images) }\end{array}$ & $\begin{array}{r}218+8 \\
\text { doubles } \\
\text { total } 234\end{array}$ & $\begin{array}{r}211+11 \\
\text { doubles } \\
\text { total } 233\end{array}$ & $\begin{array}{r}222+17 \\
\text { doubles }+ \\
4 \text { triples } \\
\text { total } 268\end{array}$ \\
\hline
\end{tabular}

Table 1. The median and interquartile range of track areas and equivalent diameters measured in SSNTDs together with the number of single circular, double, triple and total tracks detected, for each etching time investigated.

Comparing the 2 hours and 4 hours etching time detectors, we observed that there were marked differences in track area; the lower the etching time the smaller the track area. In the SSNTDs etched for between 2 and 4 hours the area of the tracks are dependent on 
etching time. We observed that there was more artefact in the 3D appearance of tracks for the 2 hours processing detector than either the 3 hours or 4 hours etching time. The smaller area at 2 hours probably results in a steeper edge angle of tracks

\section{Coalescing tracks}

In the 9 images for the two hour etching there were 8 double tracks and for the three hour etching time there were 11 double tracks. For the four hours etching time there were 17 double tracks and 4 triple coalescing tracks.

\section{Discussion.}

The rate of etching along the track must be greater than the rate of etching of the bulk material. The observed track length increases as etching takes place. As a result of surface erosion during the etching process, the length of the track could theoretically decrease in particular for angled tracks. Similarly, as the etching process continues the transverse dimensions of the track varies with processing. Hence the importance of understanding the impact of the etching process when it comes to counting tracks. Etchability of tracks will also vary depending on particle parameters such as charge, mass and velocity (Tommasino, 1997). A low-velocity particle, for example, will lose energy through low-energy collisions and the energy will be confined to a limited volume around the particle pathway (Tommasino, 1997).

The observable track etched in the above process is influenced by the critical angle of etching. Each detector - etchant combination together with etching temperature influences their angle. This then influences the detectors detection threshold - CR39 (Canada Resin- 
1939 or polyallyl di-dycol carbonate / PADC) being much more sensitive compared to Cellulose Nitrate (LR115 for example) or bisphenol-A polycarbonate (Lexan for example) (Bhagwat, 1993; Tommasino, 1997). CR39 detectors are also said to be more similar to human tissue than other similar detectors (Caresana et al., 2012), hence their selection in our study. There is in such detectors a critical angle of registration for tracks below which a track is not developed. It is important to note that through the etching process the slope of these tracks will change. A track will only have a point when it has been etched to the end of its range. With continued etching such tracks will no longer be seen.

To summarise the above, there are three inter-related parameters controlling track depth, and shape.

- Detector processing (etching solution strength, time, temperature etc.),

- the irradiation conditions,

- particle interaction with the detector.

Our data show that even at an etching time of 2 hours the microscope clearly detected a similar number of tracks to that at 4 hours etching time. However, the higher etching time was associated with more double coalescing tracks and some triple coalescing tracks that were not seen for 2 hours and 3 hours etching.

There are practical consequences to changes in processing time. For example, shorter SSNTD etching time could help improve turnaround time as well as reduce cost. Incorrect processing and reading of detectors could potentially make the difference between a property being declared 'safe' at $150 \mathrm{~Bq} \mathrm{~m}^{-3}$ (below the UK Action Level, but the UK Target Level of $100 \mathrm{~Bq} \mathrm{~m}^{-3}$ ) and it being declared as requiring remediation (that is, $200 \mathrm{~Bq} \mathrm{~m}^{-3}$ or 
above). This could result in a property being remediated when it was technically not required and vice-versa. So for any individual radon risk assessment 'accuracy' (and interpretation) of results is key.

\section{Conclusions.}

If there is too little etching, track sizes would be reduced and hence could be more difficult to image; thus there is a balance required to obtain suitable measurement accuracy. The smaller area at 2 hours processing probably results in a steeper edge angle of tracks which accounts for the greater amount of artefact in the 3D images.

Lastly, the similar number of tracks seen with the detectors in this study confirms the suitability of our methodology, employed in for example Gillmore et al., (2012) when assessing the radon outputs from radium painted watch dials.

The degree to which etching may prevent visualising adjacent tracks requires further study as it is possible that etching could result in some tracks being subsumed in other tracks as suggested in particular by the coalescing tracks numbers in our study.

Our study has shown that modern microscope imaging can detect the smaller size tracks? seen for example at 3 hours and shorter etching times may result in fewer coalescing tracks as each track has a lower diameter. Thus this study suggests that etching for periods less than four hours merits further investigation.

\section{Acknowledgements.}


This research did not receive any specific grant from funding agencies in the public, commercial, or not-for-profit sectors. However it did form part of the output from the UNESCO IUGS International Geoscience Project (IGCP) 571 on 'Radon, Health and Natural Hazards'.

\section{References.}

Bhagwat, A.M., 1993. Solid State Nuclear Track Detection: Theory and Applications. Indian Society for Radiation Physics, Kalpakkam Chapter, ISRP(K)-TD-2, 34pp.

Caresana, M., Ferrarini, M., Fuerstner, M., Mayer, S., 2012. Determination of LET in PADC detectors through the measurement of track parameters. Nuclear Instruments and Methods, Phys Res A., 683, 8-15.

Darby, S., Hill, D., Auvinen, A., Barros-Dios, J.M., Baysson, H., Bochicchio, F., Deo, H., Falk, R., Forastiere, F., Hakama, M., Heid, I., Kreienbrock, L., Kreuzer, M., Lagarde, F., Mäkeläinen, I., Muirhead, C., Oberaigner, W., Pershagen, G., Ruano-Ravina, A., Ruosteenoja, E., Schaffrath Rosario, A., Tirmarche, M., Tomásek, L., Whitley, E., Wichmann, H.E., Doll, R., 2005. Radon in homes and risk of lung cancer : collaborative analysis of individual data from 13 European case-control studies. British Medical Journal 330, 223-226.

Dörschel, B., Hartmann, H., Kadner, K., 1996. Variations of the Track Etch Rates along the Alpha Particle Trajectories in Two Types of CR-39. Radiation Measurements 26, 51-57.

Dörschel, B., Bretschneider, R., Hermsdorf, D., Kadner, K., Kühne, H., 1997. Measurement of the track etch rates along proton and alpha particle trajectories in CR-39 and calculation of the detector efficiency. Radiation Measurements 31, 103-108. 
Durrani, S., 1997. Alpha-Particle Etched Track Detectors: In Durrani, S., Ilic, R. (eds.), Radon measurements by etched track detectors. Applications on Radiation Protection, Earth Sciences and the Environment. World Scientific, Singapore, p.77-101.

Gillmore, G.K., Crockett, R., Denman, T., Flowers, A., Harris, R., 2012. Radium dial watches, a potentially hazardous legacy? Environment International 45, 91-98.

Gray, A., Read, S., McGale, P., Darby, S. 2009. Lung cancer deaths from indoor radon and the cost effectiveness and potential of policies to reduce them. British Medical Journal 2009, 338, a3110.

Mozzo, P., Trotti, F., Temporin, A., Lancai, M., Predicatori, F., Righetti, F., Tacconi, A., 1996. $\alpha$-spectroscopy on CR39 Track Detectors for the Dosimetry of Radon Daughters. Environment International 22(1), S595-600.

Ng, F.M.F., Lik, K.Y., Nikezic, D., Yu, K.N., 2007. Determination of alpha-particle track depths in CR-39 detector from their cross-sections and replica heights. Nuclear Instruments and Methods in Physics Research B, doi: 10.1016/j.nimb.2007.04.147.

Nikezic, D., Yu, K.N., 2015. Theoretical feasibility study on neutron spectrometry with the polallyldiglycol carbanote (PADC) solid-state nuclear track detector. Nuclear Instruments and Methods A 771, 134-138.

Palacios, F., Palacios Fernandez, D., Ricardo, J., Palacios, G.F., Sajo-Bohus, I., Goncalves, E., Valin, J.L., Monroy, F.A., 2011. 3D nuclear track analysis by digital holographic microscopy. Radiation Measurements 46, 98-103. 
Phillips, P.S., Denman, A.R., Crockett, R.G.M., Gillmore, G., Groves-Kirkby, C.J., Woolridge, A., 2004. Comparative Analysis of Weekly vs. Three monthly radon measurements in dwellings. DEFRA Report No., DEFRA/RAS/03.006.

Smilgys, B., Guedes, S., Morales, M., Alvarez, F., Hadler, J.C., Coelho, P.R.P., Squeira, P.T.D., Alencar, I., Soares, C.J., Curvo, E.A.C., 2013. Boron thin films and CR39-detectors in BNCT: A method to measure the ${ }^{10} B(n, \alpha)^{7}$ Li reaction rate. Radiation Measurements $50,181-186$.

Tommasino, L., 1997. Track Registration: Etching and Counting Methods for Nuclear Tracks. In Durrani, S., Ilic, R. (eds.), Radon measurements by etched track detectors. Applications on Radiation Protection, Earth Sciences and the Environment. World Scientific, Singapore, p.129-141.

Tommasino, L., 2004. Detectors/Dosemeters of galactic and solar cosmic rays. Radiation Protection Dosimetry 109, 365-374.

Wertheim, D., Gillmore, G., Brown, L., Petford, N., 2010a. A new method of imaging particle tracks in Solid State Nuclear Track Detectors. Journal of Microscopy 237, 1-6.

Wertheim, D., Gillmore, G., Brown, L., Petford, N., 2010b. 3-D imaging of particle tracks in solid state nuclear track detectors. Natural Hazards and Earth System Sciences 10, 10331036.

Wertheim, D., Gillmore, G., 2014. Application of confocal microscopy for surface and volume imaging of solid state nuclear track detectors. Journal of Microscopy 254(1), 1365-2818. 


\section{Figures Captions.}

Figure 1. Glass desiccation chamber adapted for radon and watch experiment. The join and plug were sealed using vacuum grease. SSNTDs were spaced as evenly as possible around the watch being tested.

Figure 2. Sections from 2D stitched images (x20) of SSNTDs etched for two hours (top left), three hours (top right) and four hours (bottom).

Figure 3. Top row of three 3D images shows results for two hour etching (surface plan and rotated views). The middle row shows 3D results for a detector etched for three hours, whilst the bottom row shows four hour etched images (all taken with x50 objective). 
Figure 1.
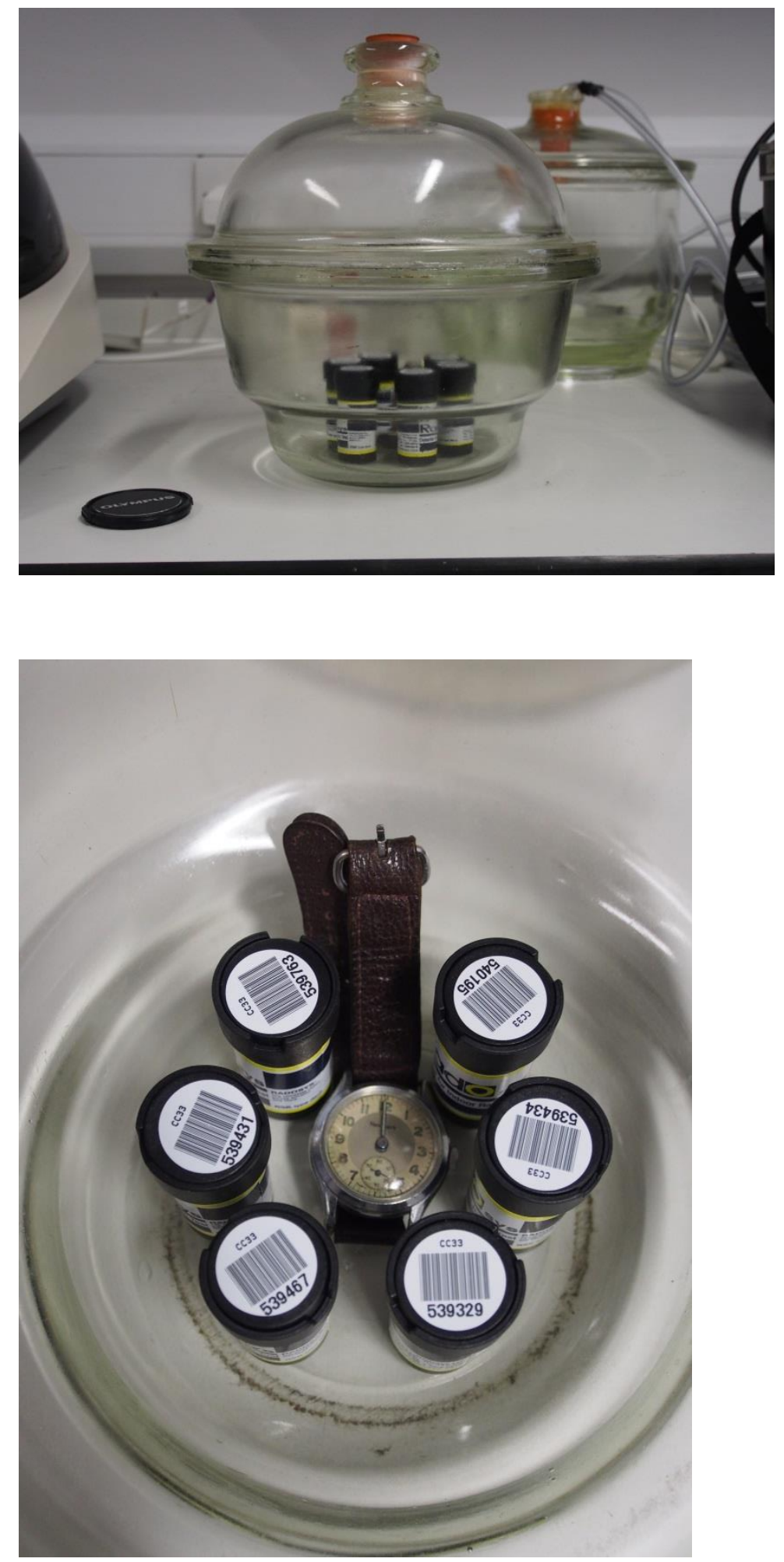

Glass desiccation chamber adapted for radon and watch experiment. The join and plug were sealed using vacuum grease. SSNTDs were spaced as evenly as possible around the watch being tested. 


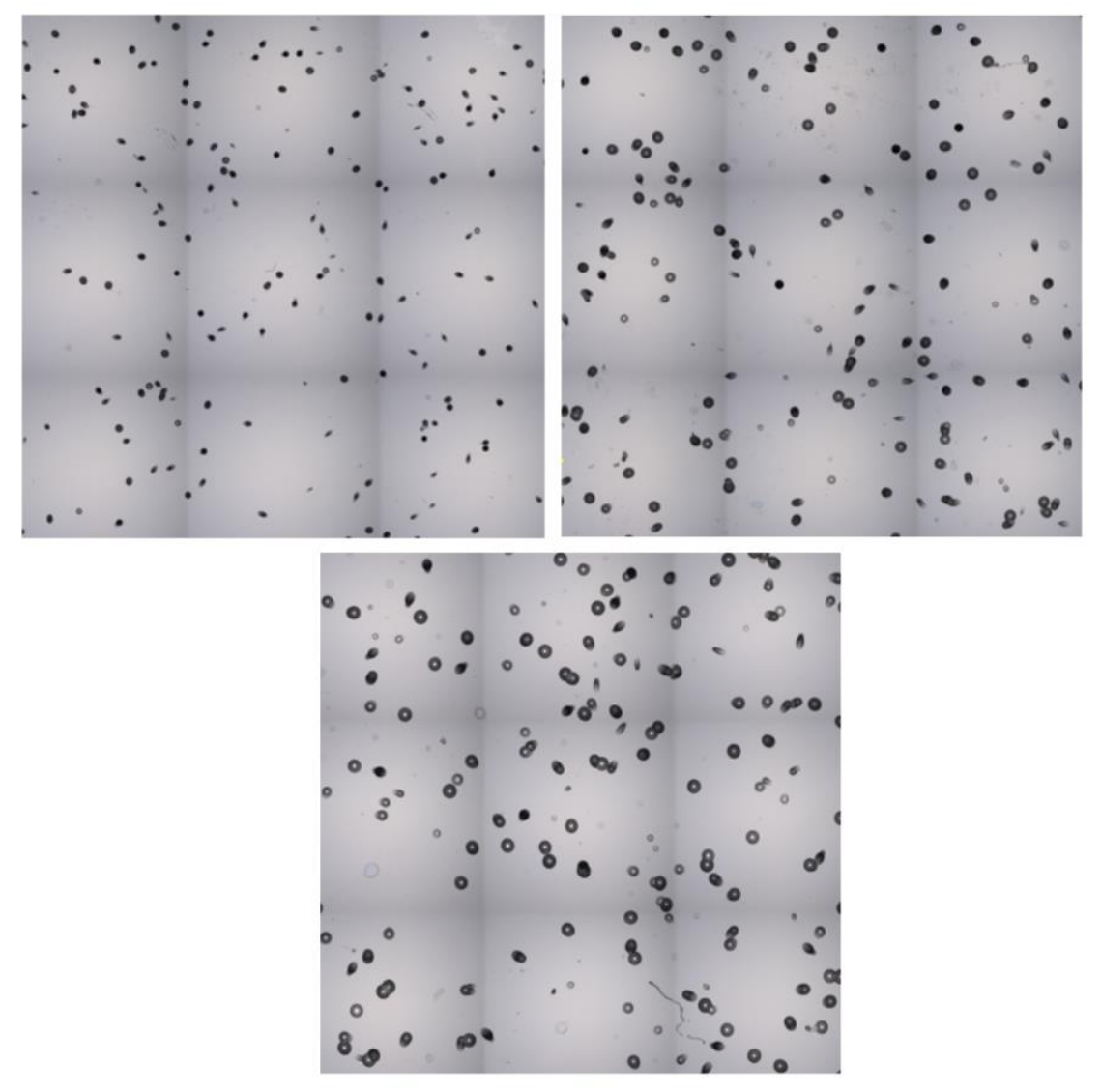



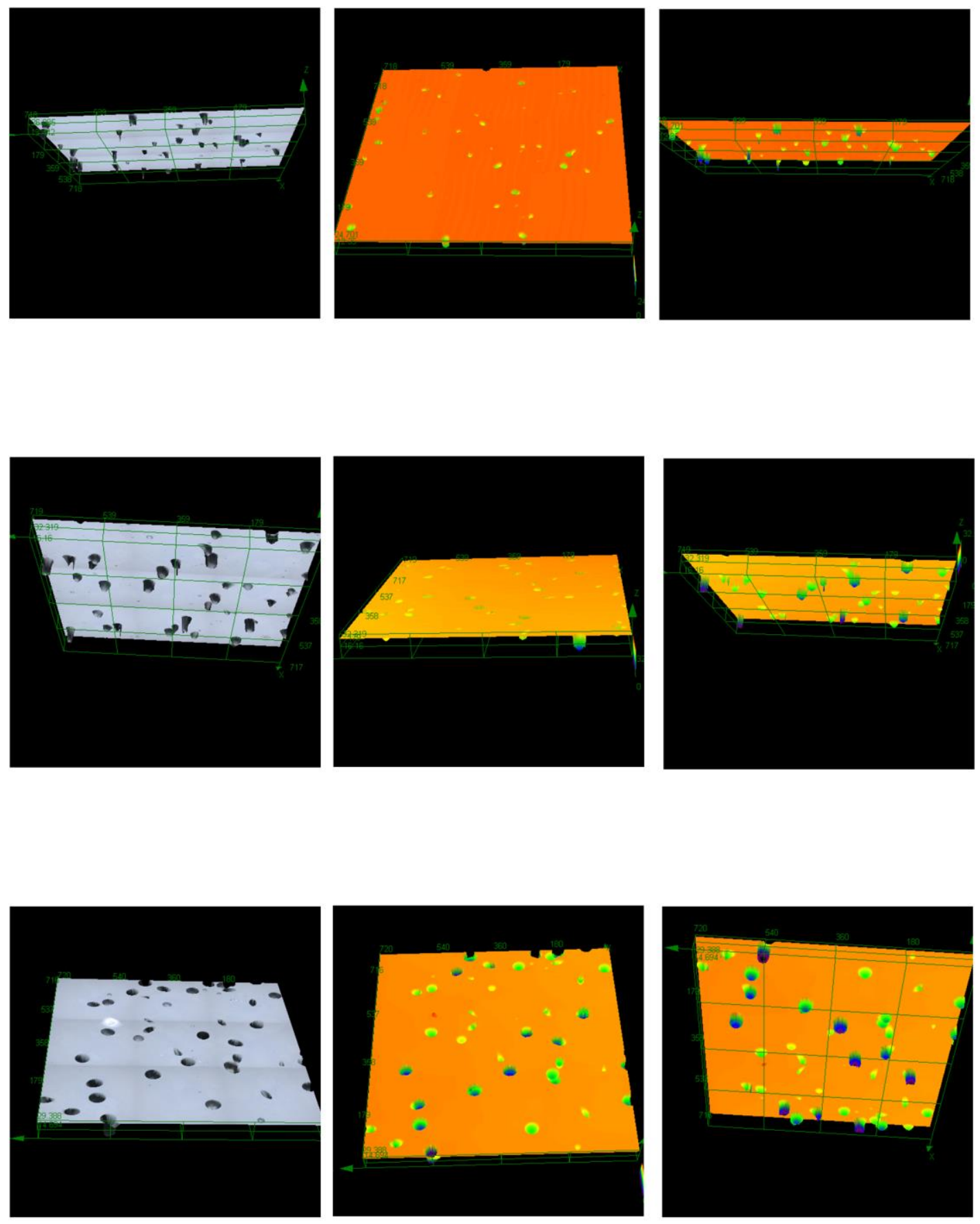
